\title{
DISCUSSION
}

\section{Towards reliable and effective site investigations}

\author{
M. B. JAKSA, J. S. GOldsworthy, G. A. FEnTON, W. S. KAGGWA, D. V. GRIFFithi, \\ Y. L. KUO and H. G. POULOS (2005). Géotechnique 55, No. 2, 109-121
}

\section{R. Baker, Technion, Israeli Institute of Technology}

The authors are to be congratulated for an interesting and informative paper. However, in the present discussion I would like to challenge one of the fundamental features of their analysis.

They characterise the spatial distribution of elastic modulus as a homogeneous random field defined by its average, coefficient of variation, and scale of fluctuation, ignoring the existence of the 'nugget effect'. The nugget effect represents fluctuations (uncertainty) on a very small scale, including also experimental errors for which the scale of fluctuation is actually zero.

The solution of the macroscopic geotechnical problem involves integration of random fields over finite volumes; the 'variance reduction function' introduced by Vanmarcke (1983) guarantees that the contribution of small-scale fluctuations cancels out, and it does not affect the solution of the stochastic boundary value problem. In their numerical simulation procedure the authors add experimental errors to the fluctuations resulting from soil variability. As their elements are rather large $(0.5 \mathrm{~m} \times 0.5 \mathrm{~m} \times 0.5 \mathrm{~m})$, such addition implies that experimental errors are perfectly correlated inside each element, and the contribution of the variability associated with small-scale fluctuation is not automatically eliminated. This process is very conservative, increasing the standard deviation of the calculated settlements.

Baecher (1986) presented a procedure making it possible to identify the magnitude of the nugget effect of various site investigation procedures, and showed that for SPT testing this effect is responsible for about half of the total experimental variability.

In summary, the variability associated with the nugget effect (mainly, but not exclusively, experimental errors) should be included in the simulation process if one wants to model results of the experimental investigation, but this component should be ignored when the random field is used as an input to solution of macroscopic geotechnical problems.

\section{Authors' reply}

The authors thank the discusser for his consideration of the authors' paper and his comments. In essence, the discusser questions the way in which measurement errors were applied in the analyses performed and reported in the paper. In particular, the discusser suggests that adoption of a nugget effect, similar to that proposed by Baecher (1982, 1986), is preferable.

The nugget effect is the manifestation of three separate phenomena (Rendu, 1981)

(a) microstructures within the geological material

(b) sampling or statistical errors

(c) measurement errors.

The discusser's comments focus on the last phenomenon. However, it is important to emphasise that the nugget effect is a combination of all three. The authors published a paper (Jaksa et al., 1997) on Baecher's technique and its relationship to the nugget effect. Jaksa et al. (1997) identified a number of important caveats associated with his approach. Most significantly, the sample spacing used to measure discrete data significantly influences the nugget and hence the random measurement error obtained from Baecher's technique. In addition, Jaksa et al. (1997) demonstrated that the nugget effect is also greatly influenced by the degree of de-trending performed on the data in order to achieve stationarity. As a consequence, the nugget effect is not associated solely with random measurement errors related to a particular test, as many authors have incorrectly assumed.

The discusser highlights results presented by Baecher (1986), which show that, for the standard penetration test (SPT), random measurement error accounts for approximately half of the variability. However, given the analyses and discussion presented by Jaksa et al. (1997), the authors suggest that the relatively coarse spacing of the SPT data sheds significant doubt on the estimated measurement error variance.

If one incorporates a nugget at the micro-scale level, the question arises as to what value to use. Very little information is available on this, especially in relation to the elastic modulus. In one of the very few studies associated with this, Jaksa et al. (2004) identified a nugget effect using the flat dilatometer test. However, given the relatively modest horizontal sample spacing of $0.5 \mathrm{~m}$, it was not possible to attribute this nugget effect solely to random measurement error, for the reasons given above and by Jaksa et al. (1997). As a starting point, the authors adopted coefficients of variation (COVs) for the random measurement error associated with the SPT and cone penetration test (CPT) of 50\% and $20 \%$ respectively. As noted by a number of researchers (for example, Lee et al., 1983; Orchant et al., 1988; Phoon and Kulhawy, 1999), these values are considered reasonable.

It is worth noting that the elastic modulus is an averaged property. This is due to the fact that settlement involves the deformation of a volume of soil. As a consequence, microvariabilities, of the type suggested by the discusser, will be locally averaged, to the point where they are negligible. Recalling that the adoption of $0.5 \mathrm{~m} \times 0.5 \mathrm{~m} \times 0.5 \mathrm{~m}$ elements was a trade-off between practical supercomputer solution times and accuracy of the results, it is expected that the errors associated with micro-variabilities are insignificant owing to local averaging.

Regarding measurement error, even if the 'point' measurement error has a COV of $50 \%$, a local average of these measurement errors over the element domain would likely lead to an average measurement error COV of about $20 \%$ (depending on the measurement error correlation structure), as was used by the authors for the case of CPT. The actual value used depends on the error variance, the true correlation structure between errors (which will probably not be white), and the local averaging domain size. As none of these things is known in practice, the way in which the measurement errors were implemented by the authors is 
entirely reasonable (and even more so in the case of SPT, where only one measurement was taken per element, given that the SPT number is an average blow count taken over a $0.45 \mathrm{~m}$ length). Regarding the possible conservatism of the analysis, it is generally deemed desirable to be on the conservative side in an engineering paper when very little is known about the magnitude and nature of measurement errors.

Finally, it is worth noting that, as both property estimation and settlement analysis involve averaging, the inclusion of measurement error has outcomes very similar to those obtained by merely increasing the random field variability and decreasing the field's scale of fluctuation - that is, the estimated field has increased variance and decreased effective scale. Again, in practice, a soil's natural variance and scale of fluctuation are generally very poorly known, so there is little to be gained by concerning oneself with the finer details of the precise nature of micro-scale variability.

\section{REFERENCES}

Baecher, G. B. (1982). Simplified geotechnical data analysis. Proceedings of the NATO Advanced Study Institute on reliability theory and its application in structural and soil mechanics, Bornholm, pp. 257-277.

Baecher, G. B. (1986). Geotechnical error analysis. Transportation Research Record, No. 1105, 23-31.

Rendu, J.-M. (1981). An introduction to geostatistical methods of mineral exploration. Johannesburg: South African Institute of Mining and Metallurgy. 